This item was submitted to Loughborough's Research Repository by the author.

Items in Figshare are protected by copyright, with all rights reserved, unless otherwise indicated.

\title{
Mesoscopic investigation of size effect in notched concrete beams: The role of fracture process zone
}

PLEASE CITE THE PUBLISHED VERSION

https://doi.org/10.1016/j.engfracmech.2019.03.028

PUBLISHER

(C) Elsevier BV

VERSION

AM (Accepted Manuscript)

\section{PUBLISHER STATEMENT}

This paper was accepted for publication in the journal Engineering Fracture Mechanics and the definitive published version is available at https://doi.org/10.1016/j.engfracmech.2019.03.028.

LICENCE

CC BY-NC-ND 4.0

\section{REPOSITORY RECORD}

Zhou, Rongxin, and Han-Mei Chen. 2019. "Mesoscopic Investigation of Size Effect in Notched Concrete Beams: The Role of Fracture Process Zone". figshare. https://hdl.handle.net/2134/38367. 


\section{the role of fracture process zone}

\section{Mesoscopic investigation of size effect in notched concrete beams:}

Rongxin Zhou ${ }^{a^{*}}$, Han-Mei Chen ${ }^{b}$

a Wolfson School of Mechanical, Electrical and Manufacturing Engineering, Loughborough University, LE11 3TU, United Kingdom

b School of Mechanical, Aerospace and Civil Engineering, University of Manchester, Manchester M13 9PL, United Kingdom

* Correspondence: r.zhou2@lboro.ac.uk

\section{Abstract}

11 A comprehensive mesoscopic investigation has been conducted into the classic topic of size 12 effect, using notched plain concrete beams subjected to three-point bending as a test bed. The concrete beams are modelled as random heterogeneous material containing three components, coarse aggregates, mortar and the interface transition zone. Mesoscopic numerical simulations using a 2D mesoscale continuum damage-based model, enhanced by a nonlocal treatment, is used to capture the whole fracture process in concrete materials. Both global and local numerical results are then examined and verified with relevant experimental evidence from the literature. A stress field interaction theory based on the strip yield model is proposed to interpret the size effect phenomenon and the role of detailed fracture process zone features is

20 discussed accordingly.

Keywords: concrete; size effect; fracture process zone; mesoscale; nonlocal 


\section{Introduction}

3 Size effect is a major issue in concrete materials and structures. Although almost all physical

4 properties, including compressive strength, tensile strength, post-peak softening and fracture

5 energy, are associated with size effect in concrete materials, in most reference publications in the literature, attention has been focused on the variation of peak load and failure strength with the specimen size [1]. In a typical definition, the size effect is the dependence of the dimensionless nominal strength $\sigma_{N}$ of a specimen on its characteristic structure size (dimension) $D$ when geometrically similar specimens are compared. The smaller the specimen size, the greater is the nominal strength.

This interesting phenomenon has been observed and under continued investigation for many years. At the beginning, it was widely believed that any experimentally observed size effect on the nominal strength of structures was of statistical origin [2], caused by randomness of local material strength and described by Weibull statistical theory based on the fact that the larger the structure the greater the probability to encounter weak points in its volume. However nowadays it gradually becomes clear that there exists another type of size effect, namely deterministic size effect [3], which exists even if exactly the same material parameters are used for different sized structures. It has also been firmly established that the size effect in quasi-brittle materials like concrete, mortar, and rocks is mainly the deterministic one [4].

As it is well known that unlike metal materials, there is a localized damage zone made of micro-cracks between the grains or through the grains around and ahead of crack tip. This localized damage zone which is also known as fracture process zone (FPZ) is deemed to have a direct relation with the macro fracture behaviour of concrete. Some researchers also concluded that the existence of the FPZ in front of a growing crack before the maximum load in concrete structure might be the intrinsic reason for the size dependence of the fracture parameters [5]. Bažant [3] proposed that the presence of sizeable FPZ at or around crack tip 
1 could lead stress redistribution within the specimen, which would result in a mismatch 2 between the size dependence of the energy release rate and the rate of energy consumption. 3 And this unbalance in the energy rates might be the source of deterministic size effect. Hu and 4 Duan [6,7] disagreed, however, and they concluded that the size effect mechanism could result from the interaction of FPZ with the nearest structure boundary.

6 Due to the important role of FPZ during fracture process in concrete, it is significant to be able to measure the size of the FPZ and on this basis to investigate the role of the FPZ in the size dependence of fracture parameters. Recently various measurement techniques are already employed to track the fracture process in concrete experimentally, for example the holographic interferometry method [8], the scanning electron microscopy method $[9,10]$, acoustic emission (AE) techniques [11] and digital image correction (DIC) method [12-16]. Among all the experimental methods used to detect the fracture process zone, the DIC is adopted mostly. In the DIC method, the fracture process zone is measured by a crack opening profile. Using the displacement field data, the crack opening at various locations of a crack is measured. The length of FPZ is determined as the distance from the notch tip to the location where the crack opening displacement (COD) becomes approximately equal to zero i.e. no damage.

From the perspective of the numerical simulation of fracture process zone, various methods have also been used. Veselý and Frantík [17] outlined a technique for estimation of the size and shape of an inelastic zone evolving around a crack tip during the tensile failure of structures made of concrete-like materials. They concluded that the shape and size of the damage zone, which referred to an inelastic zone in their mode, agree well with the fracture process zone measured by $\mathrm{AE}$ (acoustic emission) from the experiments. Grassl et al.[18] analysed the size effect on fracture process zone in notched and un-notched beams under three points bending with a lattice model at meso-scale. The fracture process zone of concrete is determined numerically by evaluating the average of spatial distribution energy densities from the resulting damage patterns of the lattice elements. Tejchman et al. $[12,19,20]$ performed 
1 several mesoscopic simulations to investigate the fracture process of notched concrete beams 2 subjected to three-point bending. The FPZ was depicted as a localized damage zone with a 3 nonlocal strain softening damage constitutive model. The influences of meso-structure such as 4 aggregate distribution, aggregate size, bond thickness etc. on the shape and size of FPZ were discussed. Numerical results were also compared with experimental evidence measured by the DIC technique. Inspired by the method used in DIC experiments, Alam et al. [21] defined the fracture process zone according to the crack opening profiles which are calculated as the relative (horizontal) displacement field between the nodes of the finite element mesh situated on either side of the localized damage zone. However, given the complexity of the internal structure of concrete, the debate is still ongoing as 'How to exactly define FPZ and further measure the size of FPZ', 'Whether size of fracture process zone can be considered as an intrinsic material property and what is the influencing physical mechanism between FPZ and the size dependent fracture parameters?'

The present study is aimed to provide some new insight into addressing the above issues and to contribute to the understanding of the role of FPZ in the size dependent nominal strength in three points bending test. A theoretic model in which a direct relationship between the FPZ and the size dependent nominal strength is proposed. In order to better approximate the real situation involving irregular aggregates, concrete is modelled as a random heterogeneous three-phase material consisting of coarse aggregates, mortar matrix and interfacial transition zone (ITZ). FPZ is defined as a localized plastic damage zone using a continuous plastic damage model enhanced by the nonlocal theory. The macro response of stress - strain curves as well as the shapes and sizes of plastic damage zones calculated from numerical results are verified against experimental observations. The influences of the specimen sizes on the shape and size of FPZ and the stress states within it are discussed in detail. Finally, it is found that the stress states interaction theory, which is proposed in present study based on the strip yield model in fracture mechanics, can be used to interpret the size dependent nominal strength observed in experiments. 


\subsection{Meso-structure generation}

3 Experimental evidences demonstrated that the mesostructure of concrete significantly

4 influence the formation and propagation of the fracture process zone [13,19]. Therefore, 5 computational modelling of concrete-like materials at mesoscale, which considers the

6 concrete compositions as coarse aggregates, mortar matrix and the interface transitional zone 7 (ITZ), is deemed to be a powerful means to describe the fracture process in concrete. In this 8 study, the mesoscale structure of concrete is represented by a stochastic distribution of 9 aggregates embedded in the mortar matrix. The aggregates are modelled by random polygon 10 particles, and the nominal size of the individual aggregates obeys a given grading curve. The 11 generation of the mesoscale geometry follows a commonly adopted take-and-place procedure 12 [22], satisfying non-overlapping and minimum gap requirements. The procedure is 13 programmed using Matlab. The density of the aggregates can be controlled by specifying a 14 volume ratio.

15 After the generation of the mesoscale structure, the geometrical data can be fed into a finite 16 element meshing processor. In the present study, ANSYS pre-processor is used, to perform the FE-meshing. Figure 1 shows the mesoscale structure of one concrete specimen (a) and its subsequent FE meshing results (b).

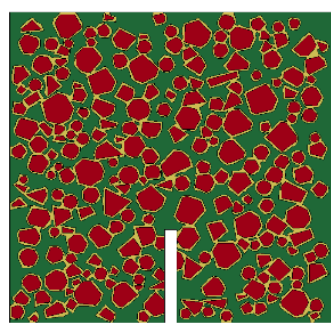

(a) Mesoscale structure

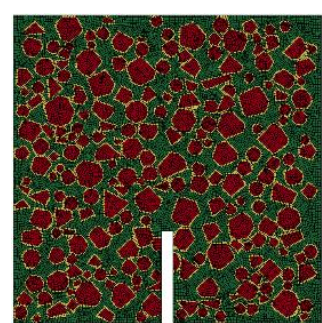

(b) FE mesh results 
1 In the generation of the mesoscale geometry, only the coarse aggregates are considered to

2 form the discrete aggregate phase. For normal concrete, the coarse aggregates include those

3 with a minimum nominal size of $4.75 \mathrm{~mm}$ [23]. Smaller aggregates are lumped into the

4 mortar phase. The volumetric ratio (or area ratio in 2D) of the coarse aggregates in present

5 study is in a range of 0.4-0.5 with the maximum aggregate size around $8 \mathrm{~mm}$.

6 It should be noted that although the current mesoscale concrete model is developed for normal

7 concrete originally, the procedure itself has no restriction on the aggregate size range, 8 aggregate shape or its distribution. Therefore, the model can be directly used to represent the 9 meso-structure of dam concrete in which much larger maximum aggregate size (around 150 $10 \mathrm{~mm}$ ) [24,25] is used. In this way, the size effect, which is a very important issue in dam 11 concrete can be further studied at mesoscale.

12 The interface transition zone (ITZ) between aggregates and mortar matrix is considered to 13 play an important role affecting the damage initiation and growth in the concrete material. 14 Therefore, in the present mesoscale model the ITZ is explicitly modelled as a separate (third) phase in the composite. Two alternative methods may be considered for the modelling of the

16 interfacial transition zone (ITZ), one using an equivalent layer of solid elements, and another using the zero-thickness cohesive plus contact interface elements as developed in [26] Preliminary analyses have indicated that, as far as the analysis of the mesoscale processes on the size effect is concerned, the use of the simpler solid element representation of the ITZ is sufficient. Therefore in the study presented in this paper, the mesoscale model employed will involve the solid element representation for the ITZ.

\subsection{Material model}

From a mesoscopic perspective, damage and fracture in concrete mainly occur in the mortar matrix and along ITZ under a general loading condition. Therefore, appropriate nonlinear material models need to be considered for these two parts in order to represent the underlying damage process. The material model employed for these two parts in the present study is the 
$1 \mathrm{~K} \& \mathrm{C}$ Concrete Damage Model. This material model is capable of describing the material

2 failure due to tension, shear, as well as compression under various stress conditions, and it 3 also includes pressure and strain rate dependent features. The

4 detailed technical information about this material model can be found in $[27,28]$. The material 5 model has been tested extensively and is found to be a suitable candidate for quasi-static as 6 well as dynamic applications of concrete-like materials [29].

7 The $\mathrm{K} \& \mathrm{C}$ concrete model uses three independent strength surfaces, namely, an initial yield 8 surface, a maximum failure surface and residual surface, with consideration of all the three 9 stress invariants $\left(I_{1}, J_{2}, J_{3}\right)$. The strength surfaces are uniformly expressed as:

$$
\Delta \sigma=\sqrt{3 J_{2}}=f\left(p, J_{2}, J_{3}\right)
$$

where $\Delta \sigma$ and $p$ denote, respectively, the principle stress difference and pressure. At any stage the current damage state is defined as a linear interpolation between the maximum and either the yielding or residual failure surface, and the interpolation factor is a function of the modified effective plastic strain measure, $\lambda$, which is defined in the following form:

$$
\lambda=\left\{\begin{array}{ll}
\int_{0}^{\bar{\varepsilon}_{p}} \frac{d \bar{\varepsilon}_{p}}{r_{f}\left(1+\left(p+\left(p / r_{f} f_{t}\right)\right)\right)^{b_{1}}} & \text { when } p \geq 0 \\
\int_{0}^{\bar{\varepsilon}_{p}} \frac{d \bar{\varepsilon}_{p}}{r_{f}\left(1+\left(p+\left(p / r_{f} f_{t}\right)\right)\right)^{b_{2}}} & \text { when } p<0
\end{array},\right.
$$

where $\bar{\varepsilon}_{p}$ is the effective plastic strain with the increment:

$$
d \bar{\varepsilon}_{p}=\sqrt{\frac{2}{3} d \varepsilon_{i j}^{p} d \varepsilon_{i j}^{p}},
$$
$b_{1}$ and $b_{2}$ are damage scaling parameters for the case of the uniaxial compression and tension, respectively; $r_{f}$ is the dynamic increase factor that account for strain rate effect, $p$ is hydrostatic pressure and $f_{t}$ is the tensile strength of the concrete material.

21 Therefore, the plastic strain output from the model represent directly the damage (cracking) 
1 pattern and can be used to describe the fracture process in concrete. This will be discussed in

2 the next subsection.

\subsection{Modelling fracture process zone}

4 In some experimental studies mentioned earlier where the DIC technique was used, the 5 fracture process zone was measured by analysing the crack opening profiles. The crack 6 opening profiles were calculated from the digital images considering the sum of the horizontal displacement of points at a certain distance on either side of the crack. More specifically in [13] the fracture process zone was defined from the notch-tip to the location where a threshold tensile strain of $83 \mu \varepsilon$ was reached. This threshold tensile strain referred to the maximum tensile strain below which concrete could still withstand stress without forming a continuous crack, and it is also known as tensile strain capacity. A similar idea is adopted in the present numerical simulation where the fracture process zone is defined as the area within which the element has already entered into softening stage. In the concrete model used in the present study, i.e. the $\mathrm{K} \& \mathrm{C}$ concrete model in LS-DYNA, the identification of this zone may be done easily with the help of the scaled damage factor $(S D F)$ that is associated with the material model. The size of the fracture process zone is then determined accordingly instead of measuring the displacement field or the stress field in the specimens.

The scaled damage factor $S D F$ is defined as:

$$
S D F=\frac{2 \lambda}{\lambda+\lambda_{m}},
$$

$\lambda_{m}$ refers to the value of $\lambda$ at the maximum failure surface. Therefore $S D F$ is in the range of $0 \sim 2$ for each independent element with $0<S D F<1$ meaning no damage, and $1 \leq S D F<2$ indicating a plastic softening process. Therefore the $S D F$ being in the range of 1 2 can be defined as the softening zone, which is equivalent to the fracture process zone. 
2 In the constitutive modelling and numerical analysis of structures made of softening materials,

3 the mesh sensitivity associated material damage is a key issue. A smeared crack band model

4 was first proposed by Bažant and Oh [30] with the assumption that cracks localize in a band

5 whose width is usually associated with a characteristic length $L_{\tilde{c}}$. For a constitutive law

6 incorporating the crack band theory to achieve a mesh independent result, three parameters,

7 namely the model I fracture energy $G_{f}^{I}$, the tensile strength $f_{t}$, and the width of the smeared

8 crack band $L_{\epsilon}$ are used to control the softening behaviour of concrete.

9 In the case where the element size in the FE model is larger than $L_{\varepsilon}$, a function of the above 10 three parameters is suggested to obtain the strain softening modulus as [30]:

$$
E_{t}=\left(\frac{1}{E}-2 \frac{G_{f}^{I}}{f_{t} L_{c}}\right)^{-1}
$$

In the case where the element size is smaller than $L_{\varepsilon}$, a fracture energy compensation

approach as expressed in Eq. (6) is adopted so that the total fracture energy consumption is preserved, assuming the failure in the local region of the FE model would be restricted to only one element width.

$$
G_{f}^{I}=h_{c} \int \sigma d \varepsilon=L_{c} \int \sigma^{*} d \varepsilon^{*}
$$

where $\sigma$ and $\varepsilon$ are the stress and strain in the FE element, and $\sigma^{*}$ and $\varepsilon^{*}$ are the nominal stress and strain over the presumed crack band width $L_{c}$. Eq. (6) indicates that the softening branch of the stress strain curve for the 'localized' element would become mesh-dependent to achieve a mesh convergent macroscopic response. This method removes the sensitivity to the mesh size in terms of the global solutions, but the strain and displacement in the local field would be mesh-dependent. This would make it difficult to evaluate the fracture process zone as it would be dependent on the chosen mesh size. 
1 Therefore, a nonlocal approach is introduced here into the numerical model to better

2 approximate the fracture process in concrete. The underlying principle of the nonlocal theory

3 is that the failure criterion of a certain point of the material is not only dependent on the stress

4 state at that point but also on the stress state in the surrounding region. Bazant [31] provided

5 supporting evidence to this argument by the observation that there exists a certain level of 6 interaction among the multiple micro-cracks within the fracture process zone. In a nonlocal

7 approach, a material characteristic length $R_{c}$ is introduced to replace a pointed local material 8 response with a weighted average over a given area to describe the stress (or strain) state of 9 the material.

10 In the present study an average weighted function based on the work by [32] is implemented.

11 If $f(x)$ is a local field in a domain $V$ then the corresponding nonlocal field is $\bar{f}(x)$ :

$$
\bar{f}(x)=\frac{\int \alpha(x, \xi) f(\xi) d \xi}{\int \alpha(x, \xi) d \xi} .
$$

13 Eq. (7) integrates within the influencing domain $V$, where $\alpha(x, \xi)$ is the weighting function:

$$
\alpha(x, \xi)=\left[1+\frac{\|x-\xi\|^{p}}{R_{c}}\right]^{-q} .
$$

The weighting function depends on the distance $r$ between the source point $\xi$ and the target point $x$. The weighting function is a monotonically decreasing function as $r$ increases, as a source point closer to the target point has a higher influence compared to the ones farther away. The material characteristic length $R_{c}$ is actually the largest interaction radius of the source point $\xi$ that affect the nonlocal average at point $x$. Thus, it restricts a representative area for the nonlocal treatment, which can be clearly interpreted in Figure 2. Unfortunately, the determination of the value for this parameter is not straightforward in concrete material due to the complex random micro-structure and the complicated failure process within it [33]. Furthermore, the value may also vary with different weighting functions. Hence in order to get an objective value for the material characteristic length for a specific numerical model, a 
1 simple but effective way would be by parameter investigation towards matching

2 representative experimental observations, which will be given in Section 3. The parameters $p$ 3 and $q$ in the weighting function (see Eq. (8)) can be set as 4 and 2 respectively as suggested in 4 [32].
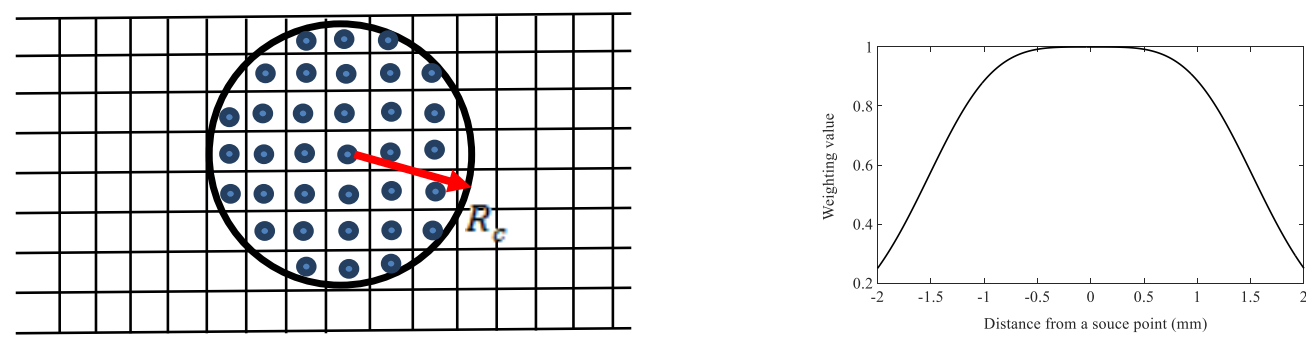

Figure 2. Nonlocal treatment and a weighting function

Theoretically, any local history variables, such as the energy release rate, strain and displacement, can be adopted for the nonlocal treatment during the calculation process. In the present study, the effective plastic strain is chosen for the weighted average during the whole process. Hence according to Eq. (7), the average effective plastic strain $\bar{\varepsilon}_{e p}$ can be written as:

$$
\bar{\varepsilon}_{e p}=\frac{\int \alpha(x, \xi) \varepsilon_{e p}(\xi) d \xi}{\int \alpha(x, \xi) d \xi},
$$

where $\varepsilon_{e p}(\xi)$ is the local effective plastic strain at a source point $\xi$. Using the effective plastic strain $\bar{\varepsilon}_{e p}$, the damage parameter $S D F$ can be calculated accordingly, from there the fracture process zone can then be evaluated.

\section{Computational framework}

For plain concrete structures subjected to bending, the nominal strength is observed to strongly depend on the size of structures. Thus three-points bending test has been the most popular apparatus in the study of concrete size effect as such a setup is also easy to operate (comparing to direct tension). In this study, the experiment conducted by Wu et al. [13], in which the fracture process evolution of concrete was investigated based on three-point 
1 bending test apparatus, is chosen for the basic numerical model setup and verification.

\subsection{Model setup for the test beams}

3 In the experiment, a series of tests were performed on the properties of FPZ in concrete using

4 the DIC technique. In the numerical model, the geometrical dimensions of the specimen are

5 set the same as the experimental case. Generally three sized concrete beams, namely $D=40$,

$6 \quad 60$ and $80 \mathrm{~mm}$ are modelled in present study. And for all the specimens, the span to height

7 ratio $S / D=4$, the thickness $B=40 \mathrm{~mm}$ and the original notch length to the height ratio

$8 a_{0} / D=0.3$ are kept as constant with the same in the experimental test The sketch of the

9 geometrical properties of the concrete beam is given in Figure 3.

10 Since the notch widths of the beam were not given in [13], a nominal value of $3 \mathrm{~mm}$ is chosen 11 in the present numerical simulations. The widths of the notches are kept the same for all specimens, similar to the treatment as some previous studies on the size effect in the

13 literatures [12,34]. To control the cost of computing time, in the finite element analysis, the 14 specimen is modelled as a plane stress problem.

15
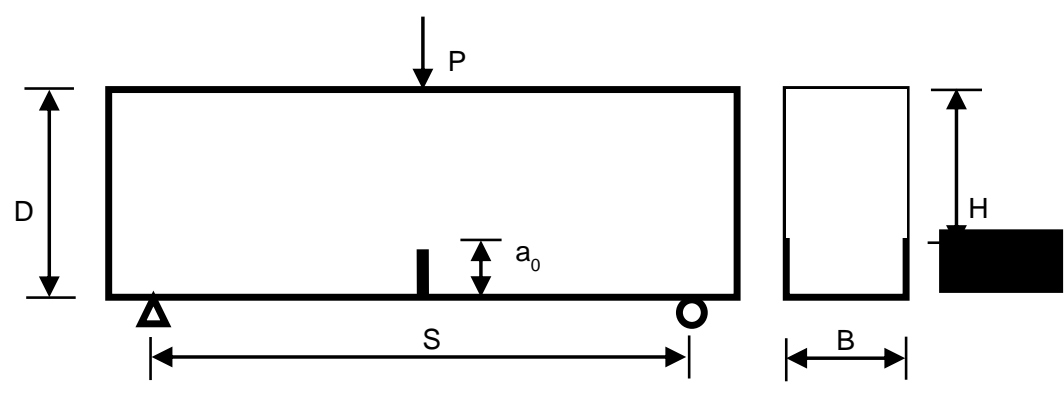

Figure 3. Specimen geometry

In modelling concrete structures such as the beam herein with a mesoscale model, two options may be considered: a) modelling the entire beam with the mesoscale model, as shown in Figure 4(a), and b) modelling only the critical region of the beam with the mesoscale model, while the remaining regions are modelled by a homogenous model, as illustrated in Figure 
1 4(b). If the critical region(s) can be identified clearly beforehand, use of the second option

2 would be effective and more efficient. Considering that the damaged zone may only localize

3 in a region close to the notch while the remaining parts are intact with no damage in a notched

4 concrete beam under three-point bending test, the meso-structure is only used in the middle

5 part of the beam to reduce the computational time. The length of the mesoscale region in the

6 middle of the beam is selected to be equal to the depth of the beam $\left(b_{m s}=D\right.$, see Figure 4$)$.

7 The remaining parts of the beams are modelled by elastic properties describing the average of

8 the elastic response of the matrix, aggregate and interfacial transition zone of the composite in

9 the meso-scale region. To demonstrate that such a modelling scheme is sound in preserving

10 the fracture behaviour of the concrete beam, direct comparisons of the beam response in terms

11 of the nominal stress vs. nominal strain relations between the full mesoscale beam model and

12 the partial (middle region) mesoscale model in a small sample beam $(D=40 \mathrm{~mm})$ are

13 presented in Figure 5. The damage zones are depicted in Figure 6. A mesh size of $0.5 \mathrm{~mm}$ is

14 chosen for both models.

15

16

17

18

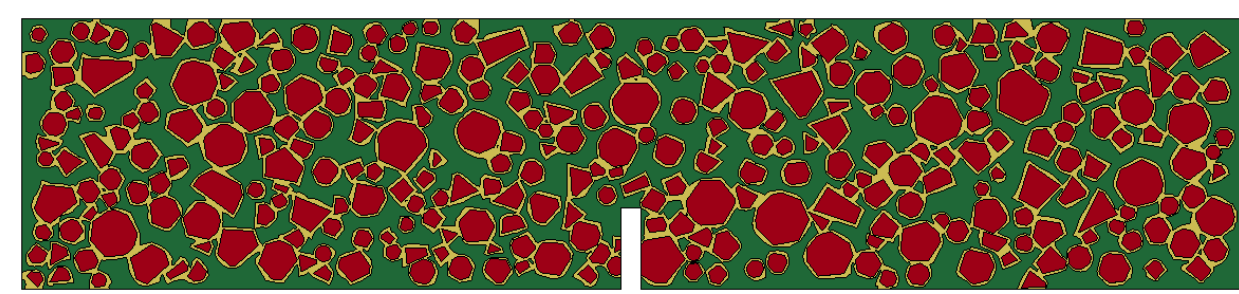

(a) Full meso-structure beam

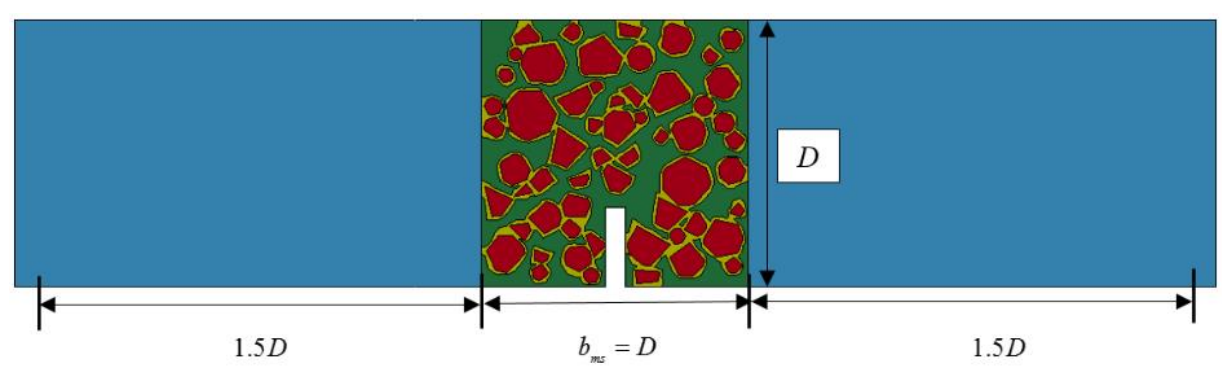

(b) Partial meso-structure beam

Figure 4. Two numerical models illustration 
1 Note that the nominal stress here for the notched beam is defined according to the elastic

2 beam theory for the ligament area above the notch without considering the stress

3 concentration factor, and by simple arrangement it can be written as:

4

$$
\sigma_{N}=\frac{1.5 P S}{B H^{2}}
$$

5 where $P$ is the applied load, $S$ is the span which has $S=4 D, B$ is the thickness of the beam,

6 and $H$ is the ligament length above the notch,

7

$$
H=D-a_{0}=0.7 D
$$

8 Therefore Eq. (10) can be further simplified as:

9

$$
\sigma_{N}=\frac{6 P}{0.49 B D}
$$

10 The nominal "strain" here is defined as the total deflection of the midpoint $u$ above the notch

11 divided by the beam height $D$, which is purely for a comparison purpose:

$$
\varepsilon_{N}=u / D
$$

13

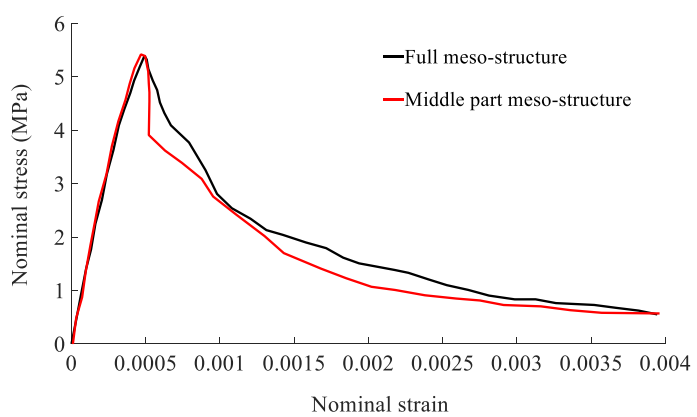

14 Figure 5. Nominal stress - strain curves for full and partial mesoscale beam models 


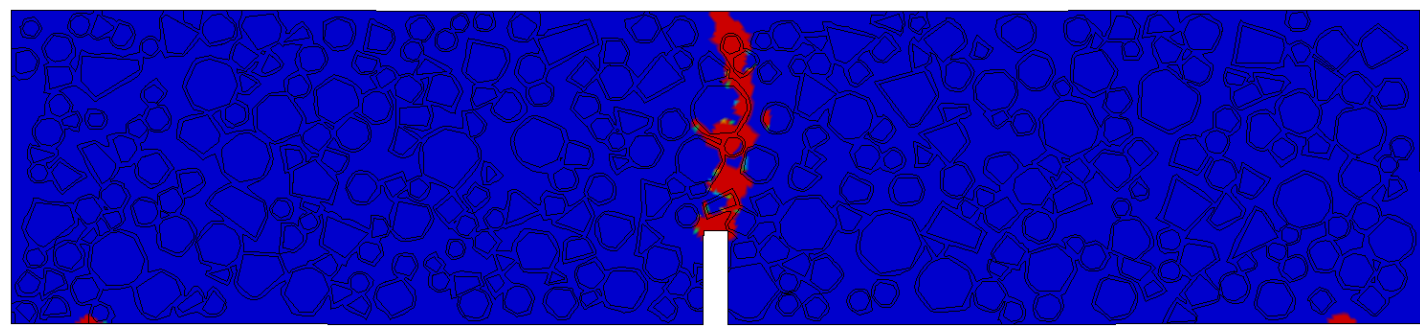

(a) Full mesoscale model

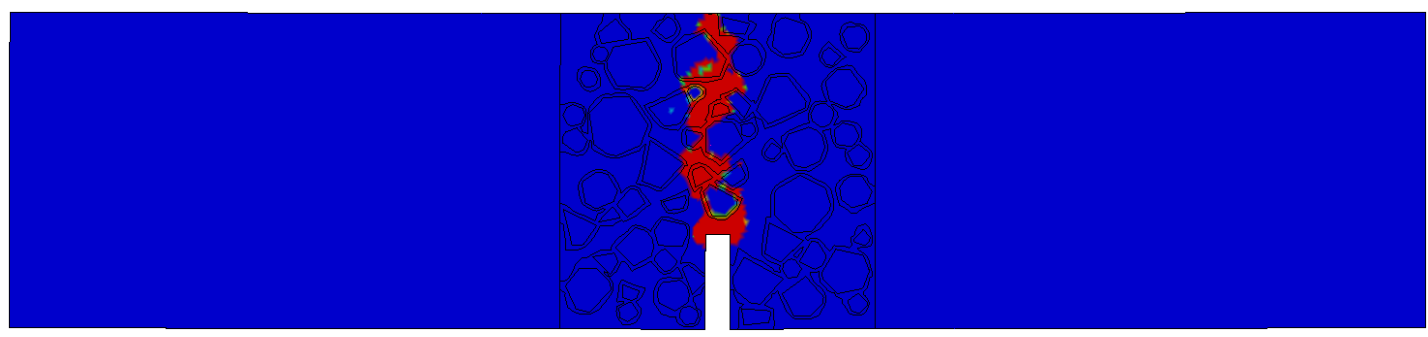

(b) Partial (middle portion) mesoscale model

Figure 6. Comparison of FPZ between the two models

6 The results show that the responses from the two beam models are essentially the same. Note

7 that some slight difference appears in the shapes of the damage zones between these two

8 models, and this is largely due to the differences in the detailed aggregare distributions in the

9 critical regions as the meso-geometry for the two models was created in two separate operations. In the subsequent analysis, the partial mesoscale modelling approach is adopted

11 for the modelling of the beam under three-point bending, with the length of the mesoscale

12 portion for the notched middle region being equal to the beam depth.

13 It should be noted here that in the present analysis we focus our attention on investigating the

14 influence of the specimen size only. For this purpose, the details of the mesoscale model itself,

15 including the aggregate volume ratio, maximum aggregate size, and the aggregate size 16 distribution are kept the same in specimens of different sizes.

As stated in Section 2, mesh dependent issue can be minimised by nonlocal treatment for both 
1 global mechanic behaviour and local fracture process. However, numerical results from the

2 nonlocal approach are actually highly dependent on the value of the characteristic length $R_{c}$,

3 and currently no deterministic value can be found for this parameter in literatures. Physically

4 the interaction radius $R_{c}$ should span a few neighbourhood elements in which the nonlocal

5 approach is implemented. However, increasing the value of the interaction radius would also

6 increase the computing time. Furthermore, when a large interaction radius is used, the

7 nonlocal influence domain will also increase which would bring in a well-known boundary

8 problem in the nonlocal approach. This boundary problem would lead to unstable

9 performance or even collapse of the calculation. Some methods have been proposed to reduce

10 such a problem [35,36], but this is beyond our current topic.

11 The effect of the material characteristic length on the evolution of the fracture process zone in the present model framework is shown in Figure 7, again using the small concrete beam $(D=$

$1340 \mathrm{~mm}$ ). As it is shown, with increasing material characteristic length, the width of the

14 fracture process zone depicted in the numerical model apparently increases. By benchmarking against the experimental observations reported in [13], which will be presented in detail in next subsection, an empirical value of $R_{c}=1.5 \mathrm{~mm}$ is adopted in present numerical

17 simulations. Note that this value is actually consistent with the suggestion on setting the material characteristic length in nonlocal approach in [19].

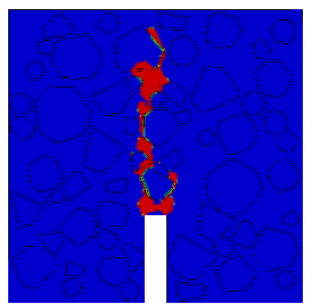

$R_{c}=0.5 \mathrm{~mm}$

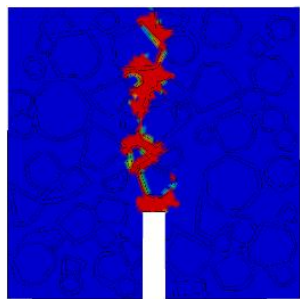

$R_{c}=1 \mathrm{~mm}$

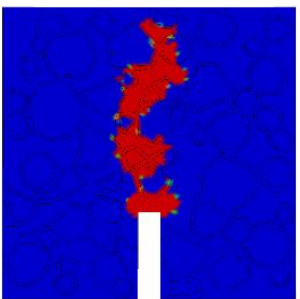

$R_{c}=1.5 \mathrm{~mm}$

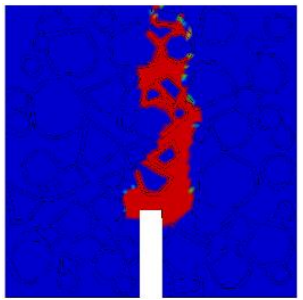

$2.000 \mathrm{e}+00$ $1.900 \mathrm{e}+00$ ] $1.800 \mathrm{e}+00$ $1.700 \mathrm{e}+00$ $1.500 \mathrm{e}+00$ $1.400 \mathrm{e}+00$ $1.300 \mathrm{e}+00$ $1.100 \mathrm{e}+00$ $1.000 \mathrm{e}+00$ 


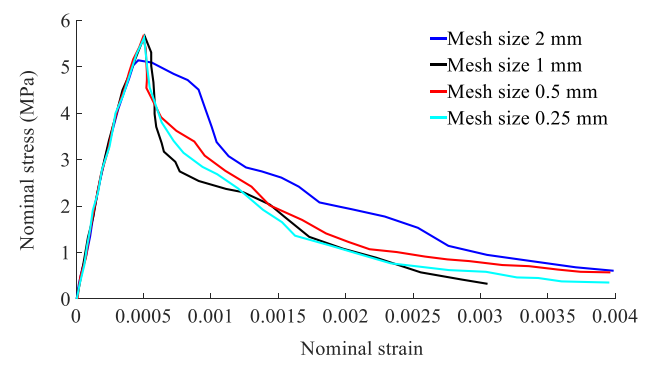

Figure 1. Nominal stress and strain curves for different mesh sizes by nonlocal treatment

3 Figure 8 shows the global response in terms of the nominal stress-strain curve for the three 4 mesh sizes $(0.25 \mathrm{~mm}, 0.5 \mathrm{~mm}$ and $1 \mathrm{~mm})$ using the nonlocal treatment with $R_{c}=1.5 \mathrm{~mm}$. The 5 corresponding fracture process zones are depicted in Figure 9. As expected, with the nonlocal 6 treatment there is negligible mesh sensitivity. The post-peak behaviour of the nominal 7 stress-strain curves in Figure 8 are virtually identical for all the mesh sizes. This ensures the 8 overall fracture energy is the same irrespective of the mesh size. Mesh independent results 9 become also apparent in the depiction of the shape and size of the fracture process zone (see Figure 9). The width of the strain localization band is almost constant for different mesh sizes.

11 The slight variation is actually due to a different number of elements involved within the 12 interaction domain, and this is deemed acceptable especially in a mesoscale model where the size and shape of individual elements inevitably varies to a certain extent. Hence, we can state that the nonlocal treatment takes a good effect here, and it benefits not only in obtaining a mesh independent global response but also in predicting the onset and evolution of the fracture process in the concrete material.

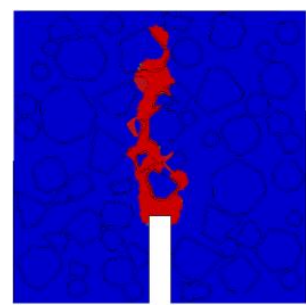

(a) mesh $0.25 \mathrm{~mm}$

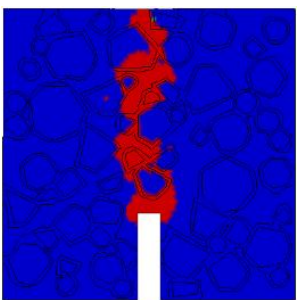

(b) mesh $0.5 \mathrm{~mm}$

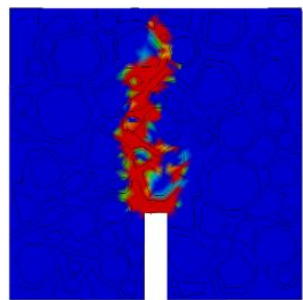

(c) mesh $1 \mathrm{~mm}$ 
3 The numerical model developed in this study is verified towards the experiments results in

4 [13], where the macroscopic responses in terms of the load versus crack mouth opening 5 displacement (CMOD) curves were given. In their experiments, load was actually the force 6 applied on the specimen while the CMOD was measured by a clip gauge installed at the centre of the notch. In the current numerical simulations, this information can be directly obtained from post-processing the simulation results (using LS-PREPOST herein).

9 Following the guidelines in Section 2.2, the material properties for the three independent components are determined firstly to match the experimental macro response of the concrete beams. The material properties for aggregates include: $E=60 \mathrm{GPa}$, mass density $\rho=2.6 \times 10^{-3} \mathrm{~g} / \mathrm{mm}^{3}$, and the properties for mortar include: $\rho=2.3 \times 10^{-3} \mathrm{~g} / \mathrm{mm}^{3}$ and compressive strength $=60 \mathrm{MPa}$. The parameters used for the ITZ include:

$14 \rho=2.3 \times 10^{-3} \mathrm{~g} / \mathrm{mm}^{3}$ and compressive strength $=40 \mathrm{MPa}$. The Poisson's ratio is assumed to be 0.2 for all the three components. Figure 10 shows a comparison of the experimental and simulated Load-CMOD curves for specimens of different sizes. A very good agreement can

17 be observed across all size groups, and this confirms that the current numerical models represent well the properties and the behaviour of the specimens used in experiment.

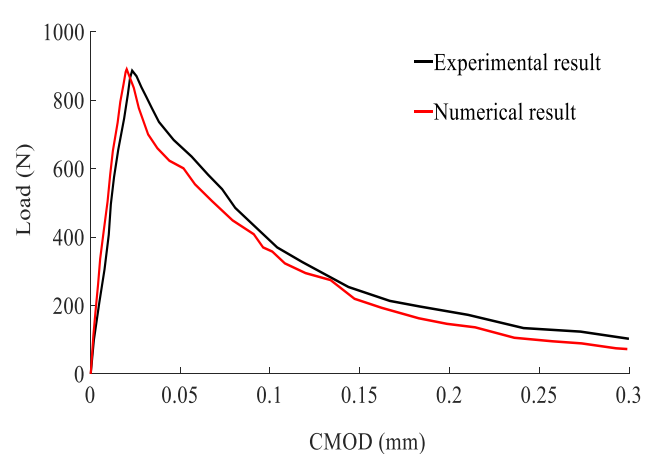

19

(a) small specimen $(D=40 \mathrm{~mm})$

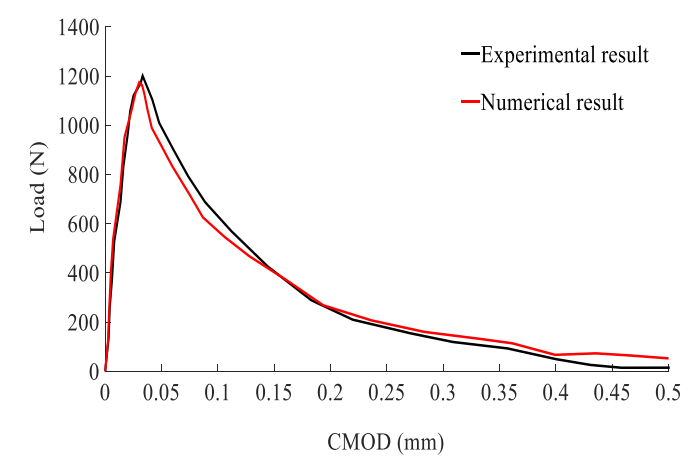

(b) medium specimen $(D=60 \mathrm{~mm})$ 


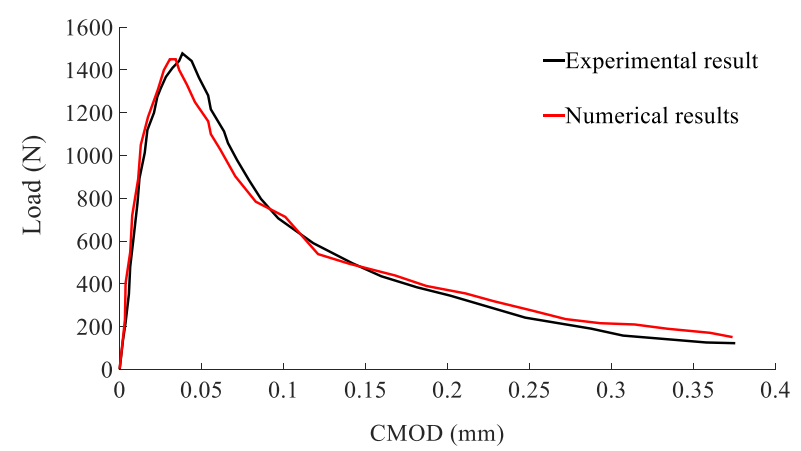

(c) large specimen $(D=80 \mathrm{~mm})$

Figure 10. Comparison of macroscopic Load-CMOD curves with experiment

5 The development processes of the damage zones defined by SDF are further checked with 6 experiemental observations in this part. Figure 11-13 illustrate the development of the fracture 7 process zone from the simulation of the specimens of three different sizes.

8

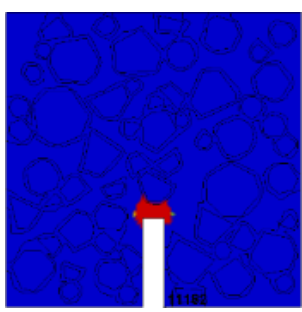

9

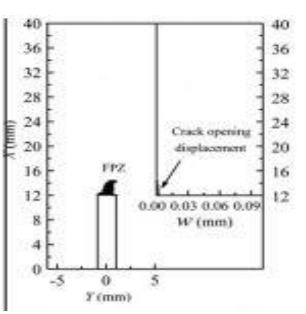

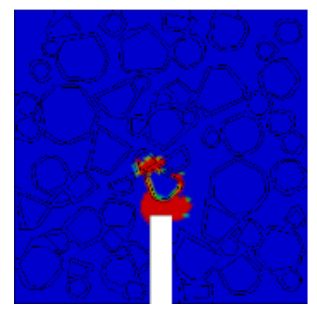

(a) Numerical results

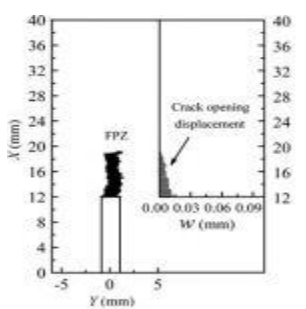

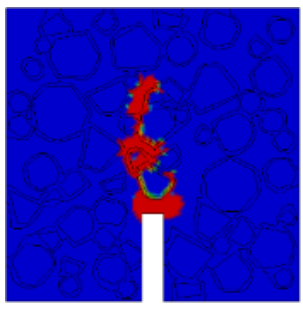

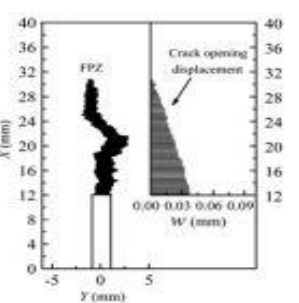

(b) Experimental results (after [13])

12 Figure 11. Development of FPZ at three loading points for small specimen $(D=40 \mathrm{~mm})$ (Left: pre-peak, 71.4\% peak load; Middle: peak load; Right: post-peak, 60.6\% peak load) 


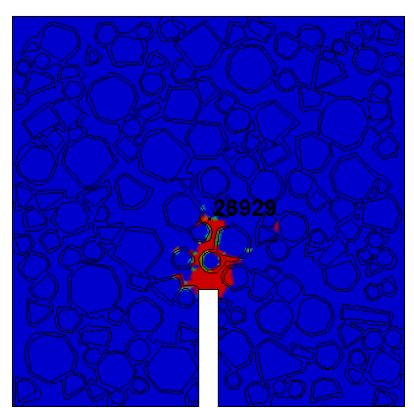

2

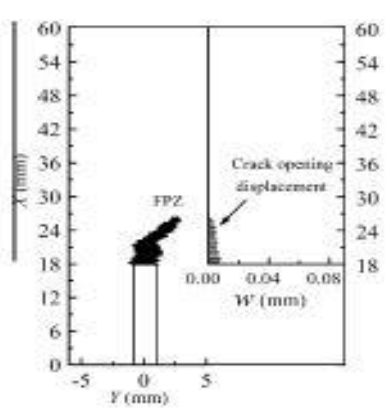

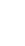

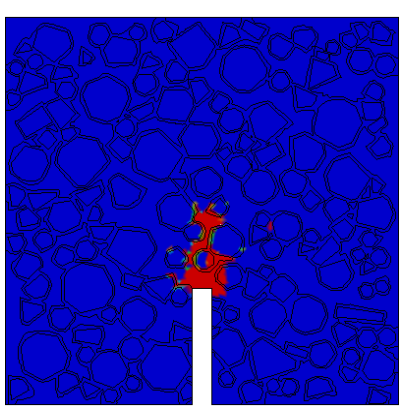

(a) Numerical nonlocal results

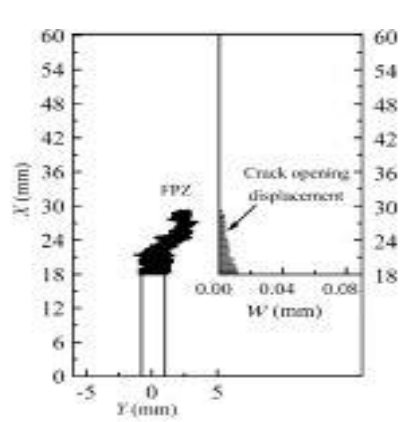

(b) Experimental results (after [13] )
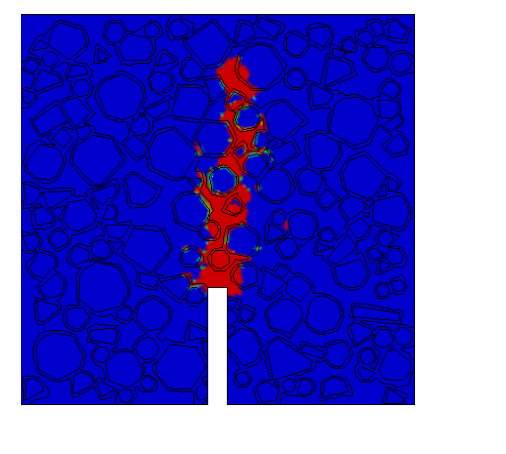

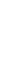
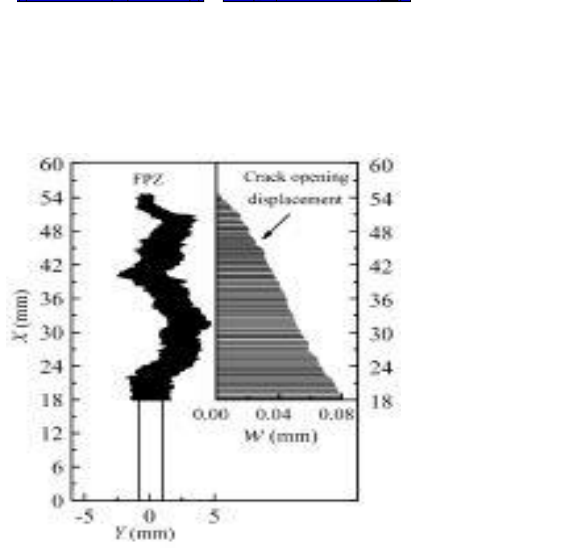

Figure 12. Development of fracture process zone at three loading points for medium specimen $(D=60 \mathrm{~mm})($ Left: pre-peak, $85.1 \%$ peak load; Middle: peak load; Right: post-peak, $40 \%$ peak load )

Apparently, the numerical results agree favourably with those from the experimental measurements using the DIC technique. Note that there appear to be some slight differences in the shapes of the highly irregular damage zones between the numerical and experimental results. These differences may be attributed to the randomness in the locations and shapes of the aggregates in the actual specimens which cannot be reproduced exactly in the numerical models. However, in terms of the measurable quantity i.e. the width and length of the damage zones, the numerical simulation results exhibit good agreement with the experimental counterparts for all the three speciments and at different loading stages. It is therefore reasonable to conclude that the present numerical model can be employed to simulate the fracture process zone for the purpose of investigating the associated size effect. 

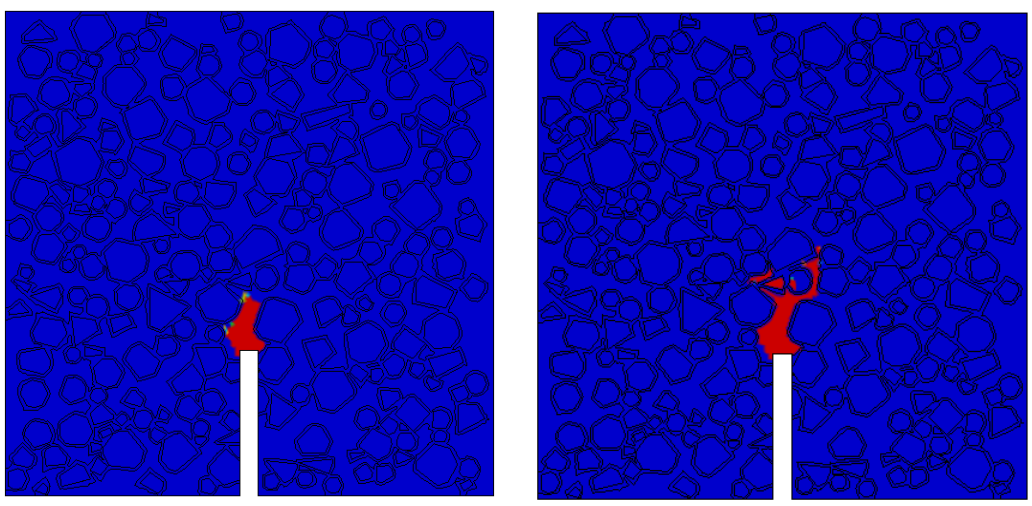

(a) Numerical nonlocal results

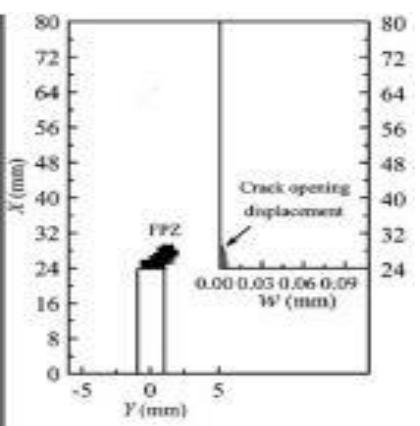

4

5 Figure 13. Development of fracture process zone at three loading points for large specimen $(D$ $6=80 \mathrm{~mm}$ ) (Left: pre-peak, 80.1\% peak load; Middle: peak load; Right: post-peak, 31.4\% peak 7 load)

\section{Results and discussion}

9 In this section the phenomena of the deterministic size effect from the numerical simulations

10 are examined. The reasons underlying the size-dependent nominal strength are discussed

11 based on the detailed numerical results. To better illustrate the tendency of the size effect, two

12 more specimens of larger sizes with $D=120 \mathrm{~mm}$ and $160 \mathrm{~mm}$, respectively, are also

13 simulated and the results are included in the discussion. All other properties except the size

14 are maintained the same in all the numerical specimens. 
2 Figure 14 shows the nominal stress and strain curves for the five different sized specimens

3 from the mesoscale concrete models. The nominal stress and strain are defined according to

4 Eq. (12) and Eq. (13), respectively. The results clearly demonstrate the so-called deterministic

5 size effect, such that the nominal strength increases persistently as the specimen size

6 decreases, and at a significant rate. Figure 15 plots the variation of the nominal strength with

7 the size of the beams. For a comparison, the variation curve based on a deterministic size

8 effect law by Bažant [37], which is valid for a structure with pre-existing notches, is also

9 enclosed wherein the nominal strength is calculated as:

$$
\sigma_{N}=\frac{K f_{t}}{\sqrt{1+\left(D / D_{0}\right)}}
$$

11 where $f_{t}$ is the tensile strength of the concrete, $D$ is the beam depth and $K, D_{0}$ are size 12 dependent parameters which are determined by fitting a set of data (with a non-linear least-square Trust-Region algorithm).

14

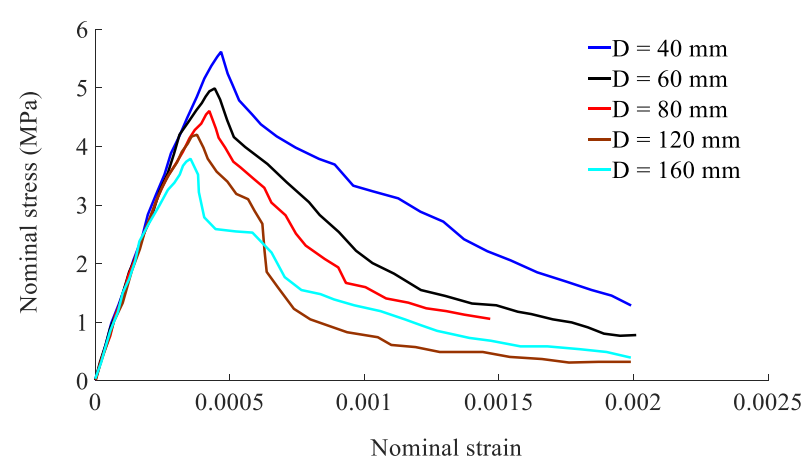

Figure 14. Nominal stress and strain curves

As it can be observed, the nominal strength of the beam from the mesoscale concrete model show a clear size dependence, and the variation matches well the size effect law proposed by Bazant [37]. In comparison, a set of simple comparative models in which the specimens are treated as entrirely homogeneous is also analysed, where the single-phased material are given the macro material properties as experiments. The material characteristic length $R_{c}$ in the 
1 nonlocal weighting function is still set at $1.5 \mathrm{~mm}$. In order to avoid any influences from the

2 meshing among the models, the comparative homogeneous models use exactly the same mesh

3 structures as their counterpart mesoscale models, except that all the elements in the

4 homogenuous models are given the uniform material properties. Clearly, the nominal strength

5 results from the homogeneous beam models show some scatter, but the overall variation trend

6 with the size can still be captured.

14

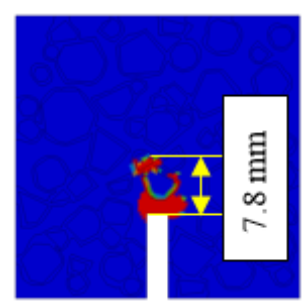

15

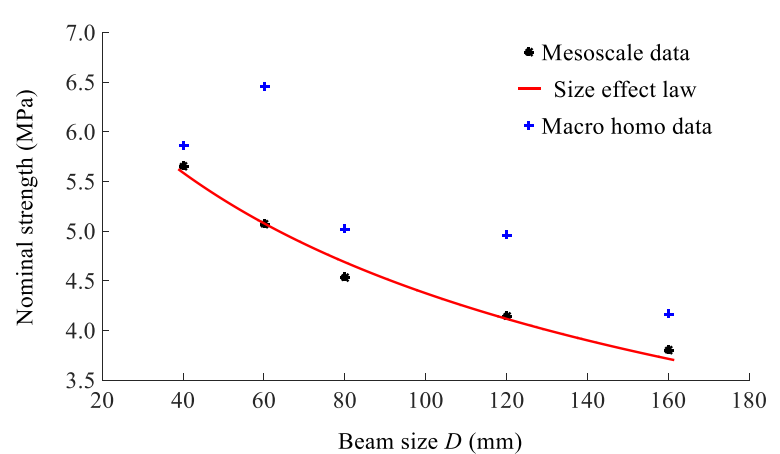

Figure 15. Nominal strength size effect tendency

\subsection{Size effect on fracture process zone}

As mentioned before, the size of the fracture process zone ahead of the notch tip before the peak loading point could be an intrinsic reason for the size effect phenomenon. Therefore it is of particular interest to examine the properties of the fracture process zone in speciemens of different sizes.

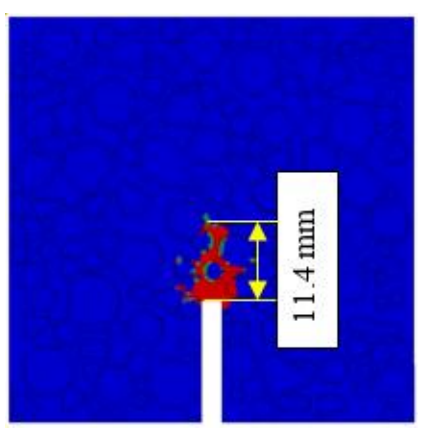

$D=60 \mathrm{~mm}$

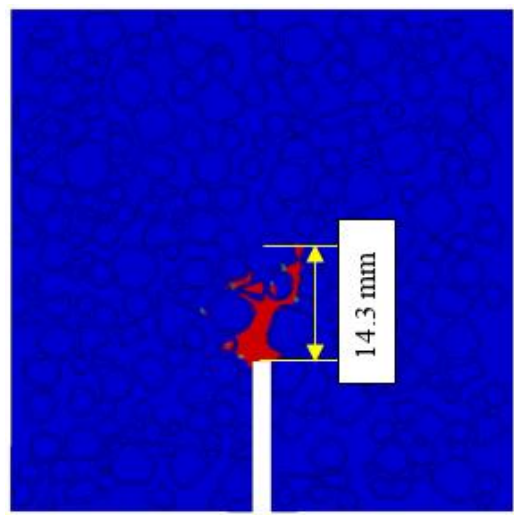

$D=80 \mathrm{~mm}$ 


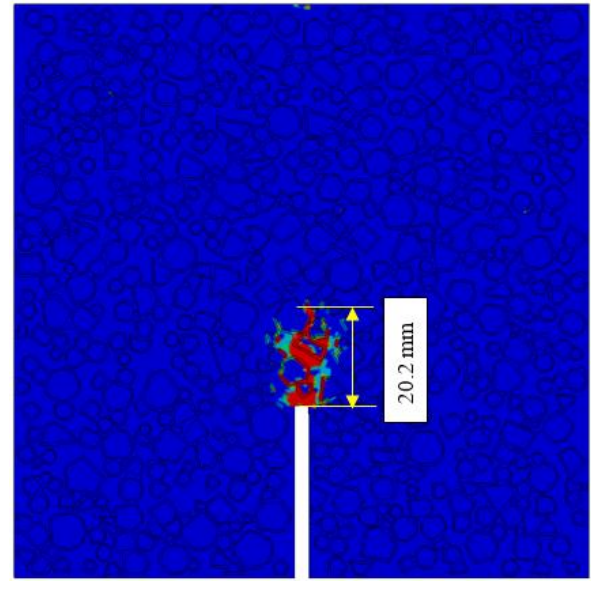

$D=120 \mathrm{~mm}$

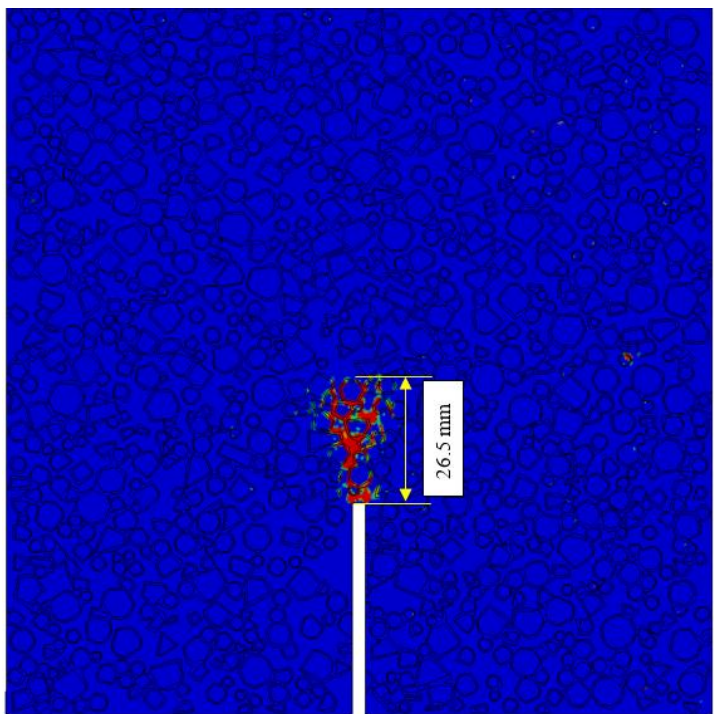

$D=160 \mathrm{~mm}$

Figure 16. The FPZs at peak load points for different specimens in mesoscale concrete model

Figure 16 shows the shapes and sizes of the fracture process zones at peak loading point for the five specimens. As can be observed, the widths of the fracture process zones are almost the same (around $5 \mathrm{~mm}$ ) for all the specimens. This phenomneon is consistent with the observations reported by Skarżyński et al. [19] using the DIC technique and previous numerical work by Grassl et al. [18]. It suggests that the width of the fracture process zone may be considered as a material property. As a matter of fact, in the crack band thoery the width of the fracture process zone is already assumed to be a constant value, and Bažant \& Oh [30] gave it three times of maximum aggregate size. However the width of the FPZ from the present numerical simulation appears to be smaller than the above assumed value. On the other hand, the absolute length of the fracture process zone at the peak loading point appears to be strongly dependent on the specimen size. Clearly, the greater the specimen size, the longer is the localized damage zone. The length increases from around $7.8 \mathrm{~mm}$ for the smallest beam $(D=40 \mathrm{~mm})$ to $26.5 \mathrm{~mm}$ for the largest beam $(D=160 \mathrm{~mm})$. This strong depdendence of the fracture zone length on the size may be explained by the decrease of stress gradient with the increase of the beam size (see Eq. (15) later). 
1 However if we further look at the relative or normalised fracture process zone length, i.e. the

2 ratio of the length of the fracture process zone to the ligament length above the notch, $l_{F P Z} / H$ 3 at the peak load, we can observe an opposite trend, such that the ratio decreases as the 4 specimen size increases. The trend is plotted in Figure 17. This result from the current 5 numerical simulation actually echoes nicely the observations made from experimental studies $6[13,16,21]$. It suggests that the normalised length of the fracture process zone is not a material 7 parameter but is dependent on the speciemen size. This size-dependent normalised length of 8 the fracture process zone provides an intrinsic explanation to the size effect which will be 9 discussed in more detail later.

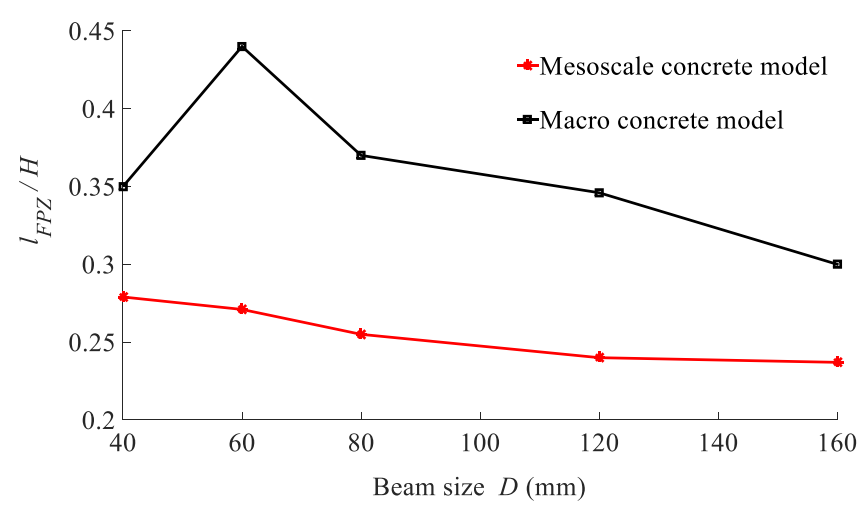

Figure 17. Relative fracture process zone length at peak load

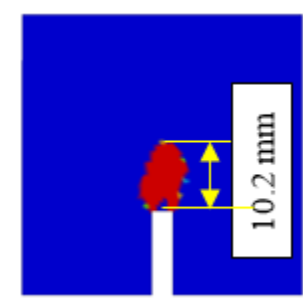

$D=40 \mathrm{~mm}$

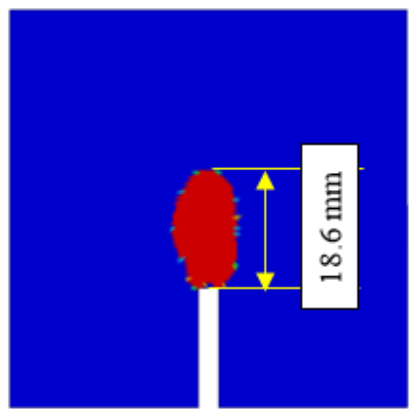

$D=60 \mathrm{~mm}$

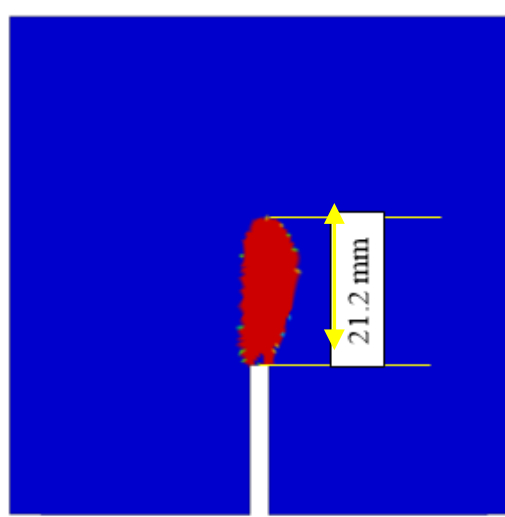

$D=80 \mathrm{~mm}$ 


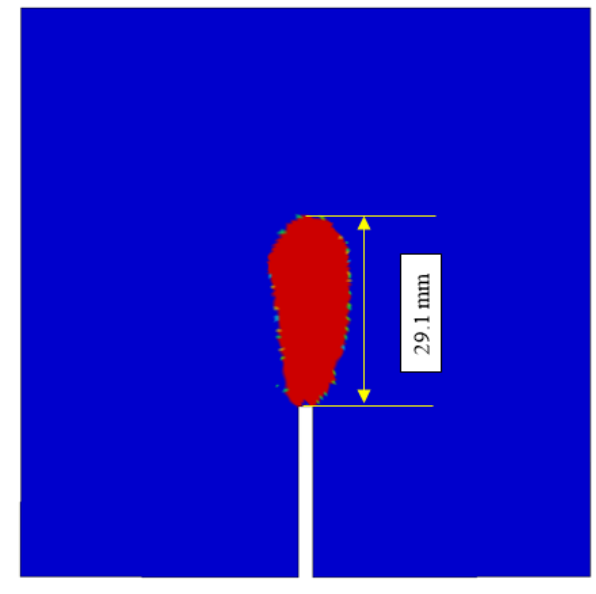

$D=120 \mathrm{~mm}$

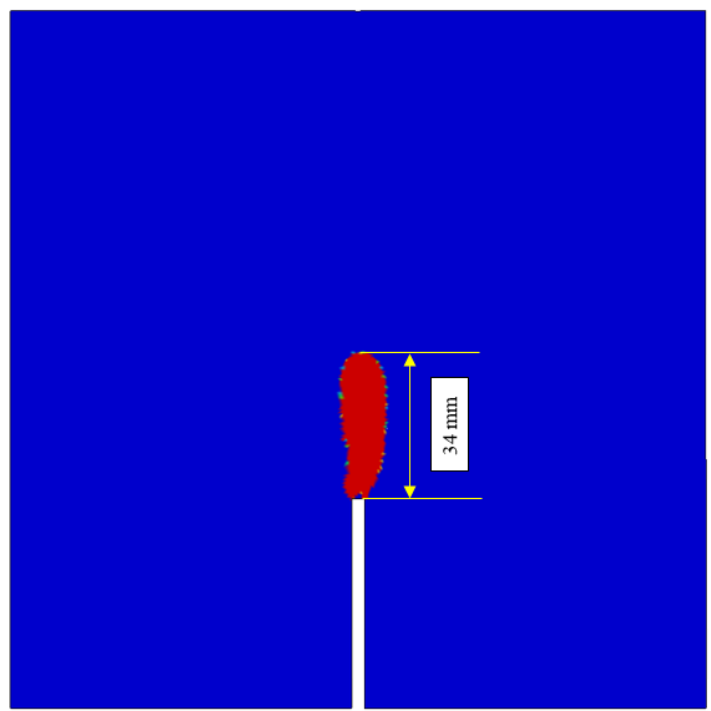

$D=160 \mathrm{~mm}$

Figure 18 The FPZs at peak load points for different specimens with homogenuous models

Figure 18 shows the caculated results in terms of the shape and size of the fracture process zone for the concrete beams of different sizes from the homogenuous concrete models. As it is shown, in contrast to the mesoscale concrete model, the evolution paths of the fracture process zones from the homogenuous models are generally straight. On the size of the fracture process zone, the general trend is similar to the mesoscale models, such that the length of the fracture process zone is strongly dependent on the size of the beam while the width is insensitive to the change of the beam size. However, the homogenuous models appear to produce longer fracture process zone lengths than the mesoscale concrete models do. The normalised fracture process zone lengths, as also shown in Figure 17, show some scatter; nevertheless the overall trend is similar to that from the mesoscale models. These observations suggest that composition of the material at the meso-scale is a significant factor that needs to be taken into account so that a more reliable evaluation of the fracture process zone may be made.

With a comparison to Figure 15, it can be found that the nominal strength variation with size is generally in line with the trend of the normalised fracture process zone length, and the 
1 scatter in the homogeneous models appear in a similar fashion. The results indicate clearly

2 that the size dependent nominal strength is directly related to the size dependent fracture

3 process zone. The scattered points on the size effect curves from the homogeneous beam

4 models suggests to be a result of lacking the ability to realistically reproduce the formation and propagation of the fracture process zone in a homogeneous model, thus affecting the detailed stress and strain states of concrete in the critical damage region.

Recent researches of size effect on dam concrete [24,25,38,39] reveal that several fracture parameters such as: fractur energy, effective fracture toughness as well as critical crack tip opening displacement, are only dependent on the specimen size within a certain range, but independent of specimen size when the ratio of the ligament height to the maximum aggregate size is large enough. However, whether the length of the fracture process zone or the normalized fracture process zone follow the same trend is not clear yet. Since the model presented in this paper has no restriction on aggregate sizes, shapes, the volume ratios, or the specimen dimensions, the loading and boundary conditions, the issue of this study should be straightforward within this model. This could be considered as future study.

As discussed above, the current mesoscale model enhanced by nonlocal treatment can well predict the size-dependent nominal strength as well as the size sensitive fracture process zone, which agree well with representative experimental evidences. However, no direct relationship between the two size-dependent factors has been established in the literature and the exact role of the size sensitive fracture process zone in contributing to the size effect on the nominal strength has not been fully understood. Herein an attempt is made to propose a theoretical model, herein referred to as a stress interaction theory, to filling in this gap and establish a quantitative relationship for the evaluation of the size effect on the nominal strength. The reference condition is still based on elastic beam bending, as adopted in the original definition of the nominal strength. So according to the elastic beam theory, there is a linear stress 
variation in a bent beam:

$$
\sigma_{E}(y)=\sigma_{L}\left(1-\frac{2 y}{H}\right)
$$

where $\sigma_{E}(y)$ is the elastic stress field along a specific cross section in the beam, $\sigma_{L}$ is the stress at the lowest fibre (bottom free surface) of this cross section, hereafter it is called the effective elastic stress, $y$ is measured from the lowest fibre where $y=0$; and $H$ is a length parameter which is related to the depth of the cross section and therefore also represents the specimen dimension. For a notched elastic beam, if the stress concentration is not considered, $H$ becomes the ligament length above the notch (Eq. (11)).

In a hypothetic situation without stress concentration and without nonlinearity in the fracture zone, the concrete beam would fail if $\sigma_{L}$ reaches the tensile strength, and consequently there would also be no size effect if the material properties are the same for the beams with similar geometrical shapes. However, in reality there exists a sizable fracture process zone, especially in concrete-like materials, within which the stress state is highly non-linear before the applied load reaching its maximum value. Herein we shall denote the non-linear stress in FPZ as $\sigma_{p}(y)$. Consequently, the equivalent elastic stress $\sigma_{L}$ deviates from the tensile strength. If $\sigma_{L}$ could be evaluated from the properties of the FPZ, that would give a direct prediction of the size effect. We postulate that the variation in the stress states within FPZ directly leads to the size dependent nominal strength, and by examining the interaction between the stress states between the elastic stress field in the absence of FPZ and the actual stress field within FPZ it could lead to an explicit correlation.

For this purpose, we adopt the strip yield model concerning fracture proposed in [40]. In the strip yield model, a long and slender plastic zone is assumed at the crack tip in a non-hardening material in a plane stress condition. The strip yield plastic zone is modelled by assuming a crack with a length of $(2 \alpha+2 \rho)$, where $\rho$ is the length of the plastic zone, with a 
1 closure stress $\sigma_{p}(y)$ applied at each tip, as shown in Figure 19. Since the stresses are finite in

2 the non-hardening materials, there cannot be a stress singularity at the new crack tip.

3 Therefore the stress intensity factors from the applied tension and closure stress cancel one

4 another,

$$
K_{\text {total }}=K_{\text {applied }}+K_{\text {closureflow }}=0
$$

6

7

8 Satisfying the above equation with the detailed formulation of the stress intensity factors, the

9 following equation can be obtained [41]:

10

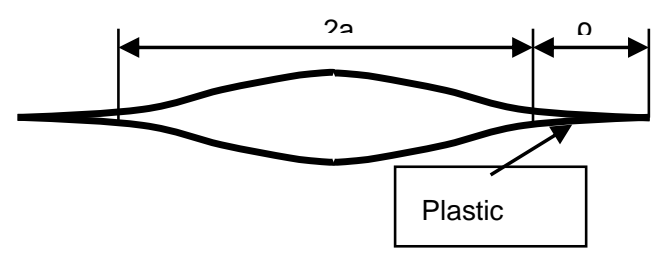

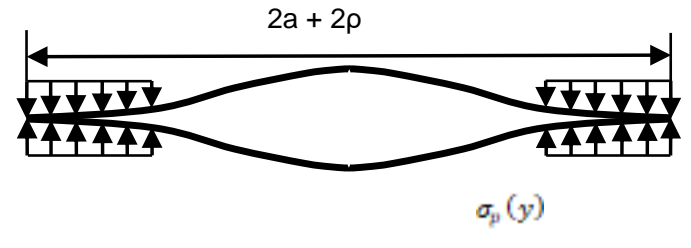

Figure 19. Sketch of strip yield model

11 where $l$ is the length of the plastic zone and $y$ is the coordinate of the point within the plastic

12 zone.

13 In numerical simulations the present stress state $\sigma_{p}(y)$ within plastic zone can be directly 14 extracted for every single element. Therefore, the integral for the plastic stress in Eq. (17) can 15 be calculated approximately by adding up all the elements within the plastic zone, i.e.:

16

$$
\int_{0}^{l} \frac{\sigma_{p}(y)}{\left(l^{2}-y^{2}\right)^{1 / 2}} d y \approx \sum_{1}^{N} \frac{\sigma_{P}(y)}{\left(l^{2}-y^{2}\right)^{1 / 2}} \Delta y,
$$

17 where $N$ is the total number of elements along the length of the plastic zone and $\Delta y$ is the

18 element mesh size adopted in the FE model. Combined with Eq. (15) and Eq. (18), Eq. (17)

19 can be transformed to: 


$$
\frac{\pi \sigma_{L}}{2}-\frac{2 l \sigma_{L}}{H}=\sum_{1}^{N} \frac{\sigma_{P}(y)}{\left(l^{2}-y^{2}\right)^{1 / 2}} \Delta y .
$$

2 The effective elastic stress can then be calculated as:

$$
\sigma_{L}=\frac{2 \sum_{1}^{N} \frac{\sigma_{P}(y)}{\left(l^{2}-y^{2}\right)^{1 / 2}} \Delta y}{\pi-4(l / H)} .
$$

4 As far as the nominal strength in concerned, this can be calculated using the effective elastic stress expressed in Eq. (20), provided the FPZ properties, including the non-linear stress field, are available at the peak loading point. If Eq. (20) can be validated as being capable of depicting the size effect tendency, it would be reasonable to deduce that the above theoretical formulation based on the interaction of the stress states theory adequately describes the intrinsic mechanism of the size effect. This will be discussed later.

\subsection{Mechanisms underlying size-dependent nominal strength}

11 In Section 4.3, an explicit relationship relating the nominal strength and the fracture process zone is established based on the concept of interaction of the stress fields. To check its validity, we can evaluate the effective stress at the peak load accordingly from the numerical simulation results.

All the required information for the calculation of the nominal stress in Eq. (20) can be easily obtained from the numerical simulation (through post processing), including the geometry parameters of each element involved as well as the stress state within the fracture process zone at peak load. The stress state $\sigma_{p}(y)$ for each $y$ position in the equation is determined as the average stress of the elements which share almost the same $y$ coordinate within the fracture process zone. The resulting nominal stress for each concrete beam calculated by Eq. (20) is presented in Figure 20. 
1 fracture process zone remains constant at the fracture strength $f_{t}$, while the fracture process

2 develops, then combining with Eq. (15), Eq. (17) can be reduced to:

$$
\frac{\pi}{2} \sigma_{L}-\frac{2 l}{H} \sigma_{L}=\frac{\pi}{2} f_{t}
$$

4 i.e.

$$
\sigma_{L}=\frac{f_{t}}{1-\frac{4}{\pi} \times \frac{l}{H}}
$$

6 Using the material property $f_{t}=3.7 \mathrm{MPa}$ and the normalized fracture process zone length in 7 Figure 17, the effective stress for beams of different sizes can be calculated. The results are 8 also included in Figure 20 for comparison.

11 It is remarkable to find that the effective stress calculated by Eq. (20) well captures the size 12 effect tendency and the effective stress value at the peak load for each beam matches very 13 closely the measured values from Eq. (12). The effective stress obtained from Eq. (22) using a The above results suggest that quantitatively it is possible to establish the trend of the size effect on the nominal strength in accordance with the stress state interaction theory proposed hypothetic elastic-perfectly plastic stress field for the FPZ indicates a similar trend but considerably under-predict the degree of the size effect in the concrete beams. in present study. The comparison with the result from involving an elastic-perfectly plastic

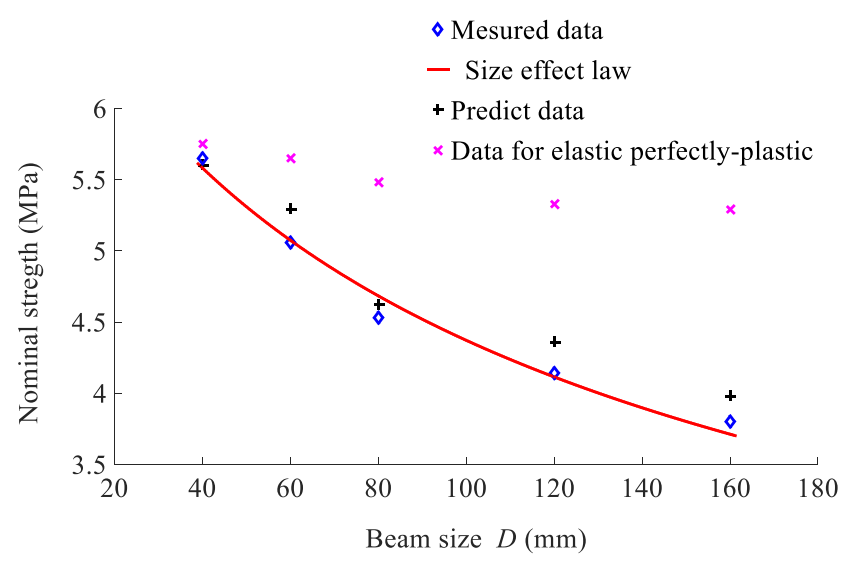

Figure 20. Size effect tendency comparison 
1 assumption in the fracture process zone provides additional insight as to why the size effect is

2 a more significant phenomenon in quasi-brittle materials like concrete and rock than in ductile

3 materials (such as steel). It also suggests that the mechanisms underlying the observed size

4 dependent nominal strength could be the combined action of the size dependent fracture process zone and the stress states interaction in the fracture process zone.

\section{Conclusions}

In this paper the classical topic of size effect in concrete structures is investigated by means of numerical simulations on geometrically similar plain concrete beams of different sizes subjected to three-point bending. The concrete beam is modelled using a mesoscale approach where three components, namely coarse aggregates, mortar and the interface transition zone are explicitly represented. A nonlocal approach is introduced to enhance the numerical model in minimising the mesh-dependent issue in the model. The numerical results are compared with relevant experimental observations using DIC technique for verification and validation purposes, and the trend of the size effect on the fracture process zone and hence the nominal strength is evaluated and discussed based on the numerical simulation results. Finally, a stress field interaction theory based on the strip yield model is proposed to establish a direct relationship between the (deterministic) size effect and the fracture process zone parameters. Based on the results, the following main conclusions may be drawn:

1.The continuum-based mesoscale concrete model, with enhancement by a nonlocal treatment, can well describe the general shape and size of the fracture process zone. The width of fracture process zone does not depend on the beam size, but the length of the fracture process zone at peak loading point is strongly dependent on the beam size, and the length of the fracture process zone increases as the beam size increases. The normalized length of fracture process zone at peak loading point, however, show an opposite trend, and in normalised terms it actually decreases with the increase of the beam sizes. 
2.The mesoscopic heterogeneity appears to have a noticeable effect on the evolution of the fracture process zone, and hence the size effect as a whole. By comparison a homogenised model lacks the ability to realistically simulate the shape and size of the fracture process zone, and this leads to an inaccurate representation of the local stress and strain states within the fracture process zone.

3. The nominal strength as produced from the mesoscale model simulation shows a good agreement with the experimental observations, and the general results confirm that the size effect is directly related to the properties of the fracture process zone at the peak load.

4.The stress field interaction theory based on the strip yield model explains very well the size effect phenomenon, and the formulation provides an explicit relationship between the size-dependent nominal strength with the length of the FPZ and the stress distribution within the FPZ.

\section{Acknowledgements}

We thank Professor Yong Lu from the University of Edinburgh for useful discussion and good suggestion in the first stage of this research. The research reported in the paper is partly funded by the Chinese Scholarship Council and the University Of Edinburgh through a joint scholarship for the $\mathrm{PhD}$ study of the first author.

\section{References}

[1] Bazant Z. Fracture and size effect in concrete and other quasibrittle materials. CRC Press 1998:616.

[2] full-text n.d.

[3] Bažant ZP. Size effect. Int J Solids Struct 2000;37:69-80. doi:10.1016/S0020-7683(99)00077-3.

[4] Morel S, Dourado N. Size effect in quasibrittle failure: Analytical model and numerical simulations using cohesive zone model. Int J Solids Struct 2011;48:1403-12. doi:10.1016/J.IJSOLSTR.2011.01.014.

[5] Zhang D, Wu K. Fracture process zone of notched three-point-bending concrete beams. Cem Concr Res 1999;29:1887-92. doi:10.1016/S0008-8846(99)00186-6.

[6] $\mathrm{Hu} \mathrm{X}$, Duan K. Size effect and quasi-brittle fracture: the role of FPZ. Int J Fract 2008;154:3-14. doi:10.1007/s10704-008-9290-7. 
1 [7] $\mathrm{Hu} \mathrm{X}$, Duan K. Mechanism behind the Size Effect Phenomenon. J Eng Mech 2010;136:60-8. doi:10.1061/(ASCE)EM.1943-7889.0000070.

[8] Regnault P, Brühwiler E. Holographic interferometry for the determination of fracture process zone in concrete. Eng Fract Mech 1990;35:29-38. doi:10.1016/0013-7944(90)90180-O.

[9] Hadjab-Souag H, Thimus J-F, Chabaat M. Detecting the fracture process zone in concrete using scanning electron microscopy and numerical modelling using the nonlocal isotropic damage model. Can J Civ Eng 2007;34:496-504. doi:10.1139/106-132.

[10] Hadjab H, Thimus JF, Chabaat M. Comparative Study of Acoustic Emission and Scanning Electron Microscope to Evaluate Fracture Process Zone in Concrete Beams. J Mater Civ Eng 2010;22:1156-63. doi:10.1061/(ASCE)MT.1943-5533.0000121.

[11] Ohno K, Uji K, Ueno A, Ohtsu M. Fracture process zone in notched concrete beam under three-point bending by acoustic emission. Constr Build Mater 2014;67:139-45. doi:10.1016/J.CONBUILDMAT.2014.05.012.

[12] Skarżyński $Ł$, Tejchman J. Calculations of fracture process zones on meso-scale in notched concrete beams subjected to three-point bending. Eur J Mech - A/Solids 2010;29:746-60. doi:10.1016/J.EUROMECHSOL.2010.02.008.

[13] Wu Z, Rong H, Zheng J, Xu F, Dong W. An experimental investigation on the FPZ properties in concrete using digital image correlation technique. Eng Fract Mech 2011;78:2978-90. doi:10.1016/J.ENGFRACMECH.2011.08.016.

[14] Alam SY, Saliba J, Loukili A. Fracture examination in concrete through combined digital image correlation and acoustic emission techniques. Constr Build Mater 2014;69:232-42. doi:10.1016/J.CONBUILDMAT.2014.07.044.

[15] Doll B, Ozer H, Rivera-Perez J, Al-Qadi IL, Lambros J. Damage zone development in heterogeneous asphalt concrete. Eng Fract Mech 2017;182:356-71. doi:10.1016/J.ENGFRACMECH.2017.06.002.

[16] Dong W, Wu Z, Zhou X, Wang N, Kastiukas G. An experimental study on crack propagation at rock-concrete interface using digital image correlation technique. Eng Fract Mech 2017;171:50-63. doi:10.1016/J.ENGFRACMECH.2016.12.003.

[17] Západočeská univerzita v Plzni V, Frantík P. Applied and computational mechanics. vol. 4. 2010.

[18] Grassl P, Grégoire D, Rojas Solano L, Pijaudier-Cabot G. Meso-scale modelling of the size effect on the fracture process zone of concrete. Int $J$ Solids Struct 2012;49:1818-27. doi:10.1016/J.IJSOLSTR.2012.03.023.

[19] Skarżyński Ł, Syroka E, Tejchman J. Measurements and Calculations of the Width of the Fracture Process Zones on the Surface of Notched Concrete Beams. Strain 2011;47:e319-32. doi:10.1111/j.1475-1305.2008.00605.x.

[20] Trawiński W, Tejchman J, Bobiński J. A three-dimensional meso-scale modelling of concrete fracture, based on cohesive elements and X-ray $\mu \mathrm{CT}$ images. Eng Fract Mech 2018;189:27-50. doi:10.1016/J.ENGFRACMECH.2017.10.003.

[21] Alam SY, Kotronis P, Loukili A. Crack propagation and size effect in concrete using a 
non-local damage model. Eng Fract Mech 2013;109:246-61. doi:10.1016/J.ENGFRACMECH.2013.07.009.

[22] Wang ZM, Kwan AKH, Chan HC. Mesoscopic study of concrete I: generation of random aggregate structure and finite element mesh. Comput Struct 1999;70:533-44. doi:10.1016/S0045-7949(98)00177-1.

[23] Wriggers P, Moftah SO. Mesoscale models for concrete: Homogenisation and damage behaviour. Finite Elem Anal Des 2006;42:623-36. doi:10.1016/J.FINEL.2005.11.008.

[24] Li Q, Guan J, Wu Z, Dong W, Zhou S. Fracture Behavior of Site-Casting Dam Concrete. ACI Mater J 2015;112:11-20. doi:10.14359/51687361.

[25] Wu Y, Xu S, Li Q, Ruiz G, Yu RC. Estimation of real fracture parameters of a dam concrete with large size aggregates through wedge splitting tests of drilled cylindrical specimens. Eng Fract Mech 2016;163:23-36. doi:10.1016/J.ENGFRACMECH.2016.06.012.

[26] Zhou R, Lu Y. A mesoscale interface approach to modelling fractures in concrete for material investigation. Constr Build Mater 2018;165:608-20. doi:10.1016/J.CONBUILDMAT.2018.01.040.

[27] Malvar LJ, Crawford JE, Wesevich JW, Simons D. A plasticity concrete material model for DYNA3D. Int J Impact Eng 1997;19:847-73. doi:10.1016/S0734-743X(97)00023-7.

[28] Tu Z, Lu Y. Evaluation of typical concrete material models used in hydrocodes for high dynamic response simulations. Int J Impact Eng 2009;36:132-46. doi:10.1016/J.IJIMPENG.2007.12.010.

[29] Zhou R, Song Z, Lu Y. 3D mesoscale finite element modelling of concrete. Comput Struct 2017;192:96-113. doi:10.1016/J.COMPSTRUC.2017.07.009.

[30] Bažant ZP, Oh BH. Crack band theory for fracture of concrete. Matériaux Constr 1983;16:155-77. doi:10.1007/BF02486267.

[31] Bažant ZP. Nonlocal Damage Theory Based on Micromechanics of Crack Interactions. J Eng Mech 1994;120:593-617. doi:10.1061/(ASCE)0733-9399(1994)120:3(593).

[32] Buste A, Lalbin X, Worswick MJ, Clarke JA, Altshuller B, Finn M, et al. Prediction of Strain Distribution in Aluminum Tailor Welded Blanks for Different Welding Techniques. Can Metall Q 2000;39:493-502. doi:10.1179/cmq.2000.39.4.493.

[33] Bažant ZP, Jirásek M. Nonlocal Integral Formulations of Plasticity and Damage: Survey of Progress. J Eng Mech 2002;128:1119-49. doi:10.1061/(ASCE)0733-9399(2002)128:11(1119).

[34] Grégoire D, Rojas-Solano LB, Pijaudier-Cabot G. Failure and size effect for notched and unnotched concrete beams. Int J Numer Anal Methods Geomech 2013;37:1434-52. doi:10.1002/nag.2180.

[35] Krayani A, Pijaudier-Cabot G, Dufour F. Boundary effect on weight function in nonlocal damage model. Eng Fract Mech 2009;76:2217-31. doi:10.1016/J.ENGFRACMECH.2009.07.007.

[36] Bažant ZP, Le J-L, Hoover CG, Bazant ZP. Nonlocal boundary layer (NBL) model: overcoming boundary condition problems in strength statistics and fracture analysis of 
quasibrittle materials 2010.

2 [37] Bazant ZP. Scaling theory for quasibrittle structural failure. Proc Natl Acad Sci U S A 2004;101:13400-7. doi:10.1073/pnas.0404096101.

[38] Guan J, Li Q, Wu Z, Zhao S, Dong W, Zhou S. Fracture parameters of site-cast dam and sieved concrete n.d. doi:10.1680/macr.15.00008.

[39] Guan J, Li Q, Wu Z, Zhao S, Dong W, Zhou S. Minimum specimen size for fracture parameters of site-casting dam concrete. Constr Build Mater 2015;93:973-82. doi:10.1016/J.CONBUILDMAT.2015.05.060.

[40] Barenblatt GI. The Mathematical Theory of Equilibrium Cracks in Brittle Fracture. Adv Appl Mech 1962;7:55-129. doi:10.1016/S0065-2156(08)70121-2.

[41] Tada H, Paris PC, Irwin GR. The Stress Analysis of Cracks Handbook, Third Edition. Three Park Avenue New York, NY 10016-5990: ASME; 2000. doi:10.1115/1.801535. 